\title{
Spatially resolved PAH emission in the inner disks of Herbig Ae/Be stars ${ }^{\star}$
}

\author{
E. Habart ${ }^{1}$, A. Natta ${ }^{1}$, L. Testi ${ }^{1}$, and M. Carbillet ${ }^{2}$ \\ 1 Osservatorio Astrofisico di Arcetri, INAF, Largo E.Fermi 5, 50125 Firenze, Italy \\ e-mail: habart@arcetri.astro.it \\ 2 Laboratoire Universitaire d'Astrophysique de Nice, UMR 6525 du CNRS, Parc Valrose, 06108 Nice Cedex 2, France
}

Received 4 March 2005 / Accepted 11 October 2005

\begin{abstract}
We present adaptive-optics high-angular resolution $\left(\sim 0.1^{\prime \prime}\right)$ spectroscopic observations in the $3 \mu \mathrm{m}$ region of eight well-known Herbig $\mathrm{Ae} / \mathrm{Be}$ stars with circumstellar disks. We detected the aromatic emission feature at $3.3 \mu \mathrm{m}$ for four out of six of our objects with flared disks (HD 169142, HD 97048, HD 100453, HD 100546), some additional features at 3.4 and $3.46 \mu \mathrm{m}$, and strong diamond features at 3.43 and $3.53 \mu \mathrm{m}$ in two of our flared objects (HD 100546 and HD 97048 respectively). We also detected hydrogen recombination line at $3.74 \mu \mathrm{m}$ in practically all the objects. The emission in the polycyclic aromatic hydrocarbon (PAHs) feature at $3.3 \mu \mathrm{m}$ and in the additional carbon dust features in the 3.4-3.5 $\mu \mathrm{m}$ region is, for the first time, spatially resolved in all the sources where the features are detected. The full-width at half-maximum sizes that we derive are typical of emission arising in a circumstellar disk. On the other hand, the continuum emission remains unresolved, with the exception of HD 97048 where it is marginally resolved. We compared the observed spatial distribution of the $3.3 \mu \mathrm{m}$ PAH feature and the adjacent continuum to the predictions of a disk model that includes transiently heated small grains and PAHs, in addition to large grains in thermal equilibrium (Habart et al. 2004a). The model predicts that, as observed, the $3.3 \mu$ m PAH emission feature is significantly broader than that of the adjacent continuum and also that about $50 \%$ of its integrated intensity comes from a radius $R<30$ AU. We find that the predicted brightness profiles reproduce the observed ones very well. This proves beyond doubt that the energetic $3.3 \mu \mathrm{m}$ PAH emission feature takes its origin in the inner disk regions.
\end{abstract}

Key words. circumstellar matter - stars: pre-main sequence - dust, extinction - radiative transfer - infrared: ISM - ISM: lines and bands

\section{Introduction}

Aromatic infrared emission bands (AIBs) attributed to PAHs have been detected in a number of pre-main sequence star of intermediate mass. According to more recent statistics based on ISO spectra, almost $60 \%$ of the Herbig Ae/Be stars (hereafter $\mathrm{HAeBe}$ ) show PAHs in emission (Acke \& van den Ancker 2004).

In Habart et al. (2004a), we investigated the presence and properties of PAHs in circumstellar disks of HAeBe stars by comparing the predictions of disk models to observations with ISO and ground-based telescopes. We showed that PAHs are both present at the surface of disks and absorb a significant fraction of the stellar radiation, similar to PAHs in the interstellar medium. Consequently, PAHs are an important source of opacity in circumstellar disks and are likely to play a dominant role in the thermal budget (via the photoelectric effect) and chemistry of the gas (via $\mathrm{H}_{2}$ formation on dust surfaces, Habart et al. 2004b). Moreover, the PAH emission is predicted to be extended, more than the emission of large grains at

* Based on observations collected at the European Southern Observatory, Chile (ESO proposal numbers: 70.C-0659, 72.C-0659). similar wavelengths. This is easily understood when considering the different excitation mechanisms of the small transiently heated particles and of the large grains at thermal equilibrium. Because of the extension of the emission, which can be more easily resolved than that of the adjacent continuum with stateof-the-art adaptive optics (AO) systems and interferometers, one can use the small particles' emission features to probe disk properties, such as the inclination of the inner disk on the line of sight.

Recently, using AO high angular resolution spectroscopic observations of the HAeBe star HD 97048, we were able to spatially resolve the emission in the diamond features at 3.43 and $3.53 \mu \mathrm{m}$ for the first time and we found that the diamond emission originates in the inner region $(R \lesssim 15$ AU) (Habart et al. $2004 \mathrm{c})$. Here we present AO high angular resolution $\left(0.1^{\prime \prime}\right)$ spectroscopic observations of a sample of HAeBe stars in the $3 \mu \mathrm{m}$ region obtained with NAOS-CONICA at the VLT. These observations allowed us to spatially resolve the inner disk emission in the PAH emission feature at $3.3 \mu \mathrm{m}$ due to $\mathrm{C}-\mathrm{H}$ bond stretching vibrations, as well as in some additional carbon dust features in the 3.4-3.5 $\mu \mathrm{m}$ region. The paper is organized as follows. Section 2 presents our sample, while Sect. 3 describes 
Table 1. Parameters of the targets. Evidence for a flared disk from spectral energy distribution modeling, infrared and millimeter interferometry observations. Presence of AIBs and silicate bands. Integrated strength of the aromatic $3.3 \mu \mathrm{m}$ feature.

\begin{tabular}{|c|c|c|c|c|c|c|c|c|c|c|c|}
\hline Object & $\begin{array}{l}d \\
{[\mathrm{pc}]}\end{array}$ & Sp. type & $\begin{array}{l}T_{\text {eff }} \\
{[\mathrm{K}]}\end{array}$ & $\begin{array}{l}L_{\star} \\
{\left[L_{\odot}\right]}\end{array}$ & $\chi^{a}$ & $\begin{array}{l}\text { Flared } \\
\text { disk }\end{array}$ & AIBs & $\begin{array}{l}I_{3.3}^{b} \\
{\left[10^{-14} \mathrm{~W} / \mathrm{m}^{2}\right]}\end{array}$ & Sil. & Group & Ref. \\
\hline HD 169142 & 145 & $\mathrm{~A} 5 \mathrm{Ve}$ & 10500 & 32 & $2.6 \times 10^{7}$ & $\sqrt{ }$ & $\sqrt{ }$ & $1(0.2)$ & - & $\mathrm{Ib}$ & (1) \\
\hline HD 97048 & 180 & B9.5Ve & 10000 & 31 & $2 \times 10^{7}$ & $\sqrt{ }$ & $\sqrt{ }$ & $1.3(0.3)$ & - & $\mathrm{Ib}$ & (2) \\
\hline HD 100453 & 114 & A9Ve & 7500 & 9 & $1.3 \times 10^{6}$ & $\sqrt{ }$ & $\sqrt{ }$ & $1.3(0.2)$ & - & $\mathrm{Ib}$ & (1) \\
\hline HD 100546 & 103 & B9Ve & 11000 & 36 & $3.6 \times 10^{7}$ & $\sqrt{ }$ & $\sqrt{ }$ & $2.5(0.5)$ & $\sqrt{ }$ & Ia & (1) \\
\hline AB Aur & 144 & B9/A0Ve & 9750 & 47 & $2.8 \times 10^{7}$ & $\sqrt{ }$ & $\sqrt{ }$ & $<1^{c}$ & $\sqrt{ }$ & Ia & (1) \\
\hline CQ Tau & 100 & F2IVe & 7500 & 5 & $7 \times 10^{5}$ & $\sqrt{ }$ & $?$ & & $?$ & I & (3) \\
\hline HD 142666 & 116 & $\mathrm{~A} 8 \mathrm{Ve}$ & 8500 & 11 & $3 \times 10^{6}$ & no & $\sqrt{ }$ & $0.3(0.2)$ & $\sqrt{ }$ & II & (1) \\
\hline HD 163296 & 122 & $\mathrm{~A} 3 \mathrm{Ve}$ & 10500 & 30 & $2.4 \times 10^{7}$ & no & - & & $\sqrt{ }$ & II & (1) \\
\hline
\end{tabular}

$\sqrt{ }$ : detection, ?: possible detection, $-:$ no detection.

${ }^{a}$ Far-ultraviolet (FUV, $6<h v<13.6 \mathrm{eV}$ ) flux at $10 \mathrm{AU}$ from the star expressed in units of the average interstellar radiation field, $1.6 \times$ $10^{-6} \mathrm{~W} \mathrm{~m}^{-2}$ (Habing 1968).

${ }^{b}$ Integrated strength (after continuum subtraction) and uncertainty (in between brackets) estimated from the ISO spectrum of Meeus et al. (2001) and Van Kerckhoven (2002).

${ }^{c}$ From Brooke et al. (1993).

References: (1) Meeus et al. (2001) and references therein; (2) Van Kerckhoven (2002) and references therein; (3) Natta et al. (2001).

the observations and the data reduction. The results are presented in Sect. 4. In Sect. 5, we compare the observations to the model predictions and Sect. 6 summarizes our results.

\section{The sample}

Our sample consists of 8 well-known HAeBe stars, all with evidence of a circumstellar disk, and observed often at different wavelengths, including ISO spectroscopy. In Table 1, we report (i) the astrophysical parameters of each star (distance, spectral type, effective temperature, luminosity, and $\chi$, i.e., farultraviolet (FUV) flux at 10 AU from the star), (ii) the observational characteristics of the disk (evidence of a flared disk), and (iii) the dust spectral characteristics (presence of AIBs and silicate bands). We also give the strength of the $3.3 \mu \mathrm{m}$ aromatic emission feature measured from the ISO spectra of Meeus et al. (2001) and Van Kerckhoven (2002).

In this sample, we can distinguish three different groups (Meeus et al. 2001): the first (group Ia) consists of stars with evidence of a flared disk and strong or moderate aromatic and silicate bands; the second (group Ib) is also characterized by flared disks and the presence of AIBs, but the silicate bands are absent; finally, in the third group (group II) there is no evidence of a flared disk, and there are weak or no AIBs, but the silicate emission features are strong.

\section{Observations and data reduction}

Long slit spectra in the L-band $(3.2-3.76 \mu \mathrm{m})$ were taken with the adaptive optics system NAOS-CONICA (NACO) at the VLT, using the visible $14 \times 14$ Shack-Hartmann wave-front sensor working at nearly $500 \mathrm{~Hz}$. The $28^{\prime \prime}$ long slit had a width of 0. '086, which roughly corresponds to the diffraction limit,

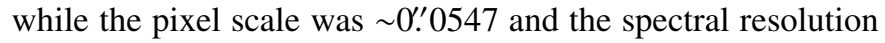

$\sim 700$. For all objects, we took one slit position centered on the star. For one of our objects, HD 97048, we took 7 slit positions: one centered on the star and the others at 0 .' $^{\prime} 043,0{ }^{\prime} 086$, $0 . ' 129$ and $-0.043,-0{ }^{\prime} 086,-0{ }^{\prime} 129$ off axis (see also Habart et al. 2004c). The long slit was aligned in the north-south direction, except for HD 100546. For HD 100546, the long slit was aligned with the major axis of the disk resolved in scattered light (Augereau et al. 2001; Grady et al. 2001) with a position angle of $\sim 160^{\circ}$ measured east of north. For each object, we employed the standard chop/nod technique with a throw of $\sim 9^{\prime \prime}$ in the north-south direction in order to correct from the atmospheric and instrumental background. The integration time per chop- or nod-positions was $1 \mathrm{~min}$, and the total integration time per source was about $30 \mathrm{~min}$.

A summary of the observations is given in Table 2. The $\log$ of the observational conditions reports Fried parameters $r_{0}$ ranging from 7 to $25 \mathrm{~cm}$ (at $500 \mathrm{~nm}$ ), average outer-scale $\mathcal{L}_{0}$ from 13 to $23 \mathrm{~m}$, and a resulting average coherent energy (after AO correction and at $2.2 \mu \mathrm{m}$ ) from 25 to $60 \%$ on axis. The last roughly gives the actual Strehl ratio characterizing the quality of our data. According to simultaneous measurements, the seeing (at $500 \mathrm{~nm}$ ) was between 0.6 and 1.5", so consistent with the NACO values. Our observations clearly benefited from good seeing conditions and AO correction, and the achieved angular resolution was close to the diffraction limit.

Data reduction was performed using a standard procedure for IR spectroscopic observations. To remove the telluric features, we used the observations of spectroscopic standard stars (listed in Table 2) taken for all sources immediately before or after the science target (except HD 97048, see Habart et al. 2004c). These reference stars that provided a good match to the objects in terms of atmospheric turbulence conditions $\left(r_{0}, \mathcal{L}_{0}\right.$, seeing) and AO correction quality were also used to estimate the point-spread function (PSF). 
Table 2. Summary of the observations.

\begin{tabular}{lllllll}
\hline \hline Object & date & $\begin{array}{l}r_{0} \\
{[\mathrm{~cm}]}\end{array}$ & $\begin{array}{l}\mathcal{L}_{0} \\
{[\mathrm{~m}]}\end{array}$ & $\begin{array}{l}\mathrm{SR}^{a} \\
{[\%]}\end{array}$ & $\begin{array}{l}\text { seeing } \\
{[\operatorname{arcsec}]}\end{array}$ & standard stars \\
\hline HD 169142 & $2004 / 03 / 12$ & 7 & 22 & 25 & 1.5 & HR 5494 \\
HD 97048 & $2003 / 01 / 11$ & 10 & 15 & 40 & 1.1 & HIP 53074, HIP 20440 \\
HD 100453 & $2004 / 01 / 30$ & 14 & 13 & 55 & 0.8 & HIP 062327 \\
HD 100546 & $2004 / 01 / 31$ & 11 & 16 & 45 & 1 & HIP 046283 \\
AB Aur & $2003 / 11 / 04$ & 11 & 23 & 30 & 0.75 & GJ1055 \\
CQ Tau & $2003 / 12 / 02$ & 13 & 20 & 45 & 0.6 & HIP025337 \\
HD 142666 & $2004 / 03 / 07$ & 21 & 22 & 60 & 0.75 & HIP 074752 \\
HD 163296 & $2004 / 03 / 15$ & 25 & 23 & 60 & 0.6 & HIP 085389 \\
\hline
\end{tabular}

${ }^{a}$ Resulting average coherent energy (after AO correction and at $2.2 \mu \mathrm{m}$ ) giving the actual Strehl ratio characterizing the quality of our data.

${ }^{b}$ The data obtained for HD 97048 has been previously presented in Habart et al. (2004c).

\section{Results}

\subsection{Spectra}

Figure 1 shows the spectra obtained for the sources in our sample integrated over the slit for distances from the star $\leq 1^{\prime \prime}$. When available, for comparison the figure also shows the spectra obtained with the Short Wavelength Spectrometer (SWS) on board ISO with a beam of $\sim 14 \times 20^{\prime \prime}$ around $3 \mu \mathrm{m}$ and published in Meeus et al. (2001) and Van Kerckhoven (2002). Both NACO and ISO spectra are normalized to the continuum emission adjacent to the $3.3 \mu \mathrm{m}$ PAH feature. The wavelength coverage of the NACO spectra allowed us to observe the $3.3 \mu \mathrm{m}$ PAH feature, some additional carbon dust features in the 3.4-3.5 $\mu \mathrm{m}$ region, and two hydrogen recombination lines at 3.297 and $3.741 \mu \mathrm{m}$, as discussed in the following.

\subsubsection{The 3.3 $\mu \mathrm{m}$ PAH feature}

In our NACO spectra, we clearly detected the $3.3 \mu \mathrm{m}$ PAH feature for four out of six of our objects in group I, whereas we do not for the two objects of group II.

For the four group I objects showing the $3.3 \mu \mathrm{m}$ feature (HD 169142, HD 97048, HD 100453, HD 100546), the feature was detected by both NACO and ISO. Comparing the NACO and ISO spectra, we found that the ratio of the emission peak to the continuum is roughly similar, i.e., that the larger ISO beam does not add more emission in the feature. This confirms that the PAH emission is confined to the disk. The exception is the ISO spectrum for HD 97048, which shows stronger PAH emission at $3.3 \mu \mathrm{m}$ than the NACO one. In this case, one could think that the surrounding nebula contributes to the emission (e.g., van Boekel et al. 2004).

The two group I objects where the $3.3 \mu \mathrm{m}$ feature is not detected are $\mathrm{AB}$ Aur and $\mathrm{CQ}$ Tau. In the case of AB Aur, the $3.3 \mu \mathrm{m}$ feature was not detected by neither NACO nor ISO. On the other hand, PAH features at 6.2, 7.7, and $8.6 \mu \mathrm{m}$ ) have been detected (Van Kerckhoven 2002; Acke \& van den Ancker 2004). The 3.3/6.2 $\mu \mathrm{m}$ ratio, as well as the $11.2(3) / 6.2 \mu \mathrm{m}$ ratio, appears anomalously low in this source (Acke \& van den Ancker 2004). One possible explanation
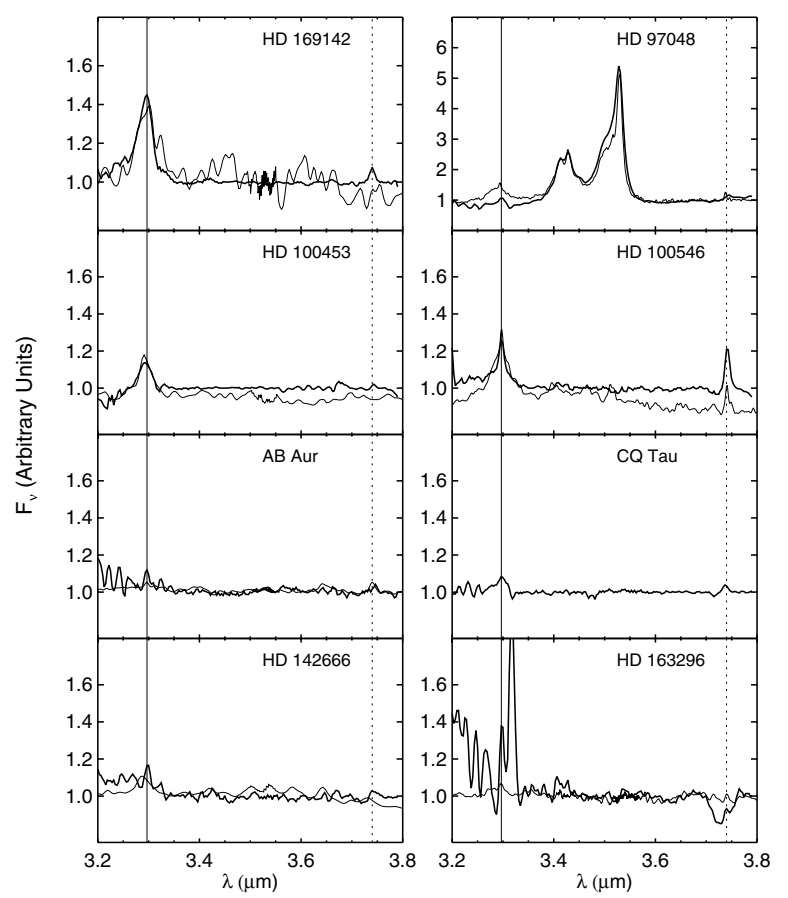

Fig. 1. NACO spectra obtained for distances from the star $\leq 1^{\prime \prime}$ (thick solid lines). The thin lines show the ISO-SWS spectrum, obtained with a beam of $14 \times 20^{\prime \prime}$. We also show the PAH feature position as solid vertical lines and as vertical dotted lines, the position of Pf $\gamma$ line at $3.74 \mu \mathrm{m}$. Both NACO and ISO spectra are normalized to the continuum emission adjacent to the $3.3 \mu \mathrm{m}$ PAH feature. For HD 163296 , the data were noisy, and it was difficult to remove the telluric features. The apparent absorption feature slightly blue-ward of the $\operatorname{Pf} \gamma$ line is due to an artefact in the data-reduction process.

could be PAH grain growth and/or ionization that will produce smaller 3.3/6.2 and 11.2(3)/6.2 $\mu \mathrm{m}$ ratios.

For CQ Tau, no ISO-SWS spectra around $3 \mu \mathrm{m}$ are available. In this system, the non detection of the $3.3 \mu \mathrm{m}$ feature may be due to low excitation conditions due to the later spectral type (F2) of the central star.

In the group II objects, an interesting case is HD 142666, which shows weak $3.3 \mu \mathrm{m}$ feature in the ISO spectra and clear evidence of the $6.2 \mu \mathrm{m}$ feature. In the NACO data, the 
signal-to-noise ratio is not sufficient to claim the presence or absence of a weak $3.3 \mu \mathrm{m}$ feature. For HD 163296, on the other hand there is certainly no strong $3.3 \mu \mathrm{m}$ feature but the data were noisy and we cannot say anything about a weak feature.

In general, the NACO spectra indicate that PAH emission has a typical disk size and that the PAH emission is strong when the disk is flared, as suggested by previous studies based on ISO data (e.g., Meeus et al. 2001; Van Kerckhoven 2002; Acke \& van den Ancker 2004; Habart et al. 2004a).

\subsubsection{The features in the $3.4-3.5 \mu \mathrm{m}$ region}

In two objects (HD 97048, HD 100546) the NACO spectra show some features in the $3.4-3.5 \mu \mathrm{m}$ region, in addition to the aromatic band at $3.3 \mu \mathrm{m}$. HD 97048 shows the peculiar strong features that peaks at 3.43 and $3.53 \mu \mathrm{m}$ (e.g., Blades \& Whittet 1980; Guillois et al. 1999; Van Kerckhoven 2002). Several studies have proposed attributing these features to surface C-H stretching modes on 10-50 nm or larger diamond particles (e.g., Guillois et al. 1999; Sheu et al. 2002; Jones et al. 2004).

Because of the good match between laboratory and observed spectra, this identification is very persuasive. As discussed in Habart et al. (2004c), we see that the diamond components are very similar in the NACO and ISO spectra in peak position, width, and relative strength. Moreover, the peak/continuum ratio in the diamond bands is roughly similar. This suggests that diamond emission is confined to the disk.

HD 100546 shows different, weaker features that peaks at 3.4 and $3.46 \mu \mathrm{m}$. In the NACO data, these features are clearly visible for distances from the star of $\sim 0.2^{\prime \prime}$ to $0.6^{\prime \prime}$ (see Fig. 4). In the ISO data, the shape of these bands is uncertain since the up and down scans differ (Van Kerckhoven 2002). These weak "satellite" bands have been observed in several PAHs sources, including reflection nebulae, planetary, or proto-planetary nebulae (e.g., Geballe et al. 1985; Jourdain De Muizon et al. 1990a,b; Joblin et al. 1996; Van Kerckhoven 2002). They could be attributed to aliphatic C-H stretches in methyl or ethyl sidegroups attached to PAHs (e.g., Joblin et al. 1996). But, these observed emission bands show some correspondences with the bands of the less-processed Orgueil nanodiamonds with mean sizes on the order of 2-3 nm (e.g., see Fig. 4 in Jones et al. 2004). The $3 \mu \mathrm{m}$ spectra of diamond surface $\mathrm{C}-\mathrm{H}$ stretching features is strongly size-dependent, and the observed meteoritic nanodiamond $\mathrm{C}-\mathrm{H}$ stretching features are in fact very different from the features seen on the surfaces of larger diamonds ( $\geq 10-50 \mathrm{~nm}$ ), as observed in HD 97048 (e.g., Sheu et al. 2002; Jones et al. 2004). Thus, these weaker "satellite" features could also arise from the emission from nanodiamonds.

\subsubsection{The hydrogen emission lines}

Finally, the observed spectral interval contains two hydrogen emission lines, namely Pf $\delta$ close to the center of the broad PAH feature at $3.3 \mu \mathrm{m}$ and Pf $\gamma$ at $3.74 \mu \mathrm{m}$. Pf $\gamma$ is visible in practically all objects and is stronger in the smaller aperture NACO data than in the ISO spectrum; its emission comes mostly from the innermost regions of the system (see Sect. 4.2.3).

In the following, we use the observed Pf $\gamma$ intensity to estimate the contribution of $\operatorname{Pf} \delta$ to the $3.3 \mu \mathrm{m}$ feature, using the theoretical intensity ratios given by Hummer \& Storey (1987). For most of our objects, we find that the contribution of $\operatorname{Pf} \delta$ to the $3.3 \mu \mathrm{m}$ feature is negligible $(\leq 4 \%)$. The exception is HD 100546, where Pf $\gamma$ is strong and Pf $\delta$ seriously affects the observed shape of the $3.3 \mu \mathrm{m}$ feature. For this object, we find that the contribution of $\operatorname{Pf} \delta$ to the $3.3 \mu \mathrm{m}$ feature is strong close to the star and decreases very rapidly in the outer parts. For distances from the star $\leq 0.1^{\prime \prime}$, the spectrum at $3.3 \mu \mathrm{m}$ is completely dominated by the contribution of $\operatorname{Pf} \delta$, we do not see the PAH emission, and the measured $\operatorname{Pf} \gamma / \operatorname{Pf} \delta$ ratio is similar to that predicted by Hummer \& Storey (1987). In the NACO spectrum for distances from the star $\leq 1^{\prime \prime}$, the contribution of $\mathrm{Pf} \delta$ to the $3.3 \mu \mathrm{m}$ feature is about $40 \%$; this contribution is lower in the ISO data, about $\lesssim 7 \%$, and the observed shape of the $3.3 \mu \mathrm{m}$ feature is less affected.

\subsection{Brightness spatial distribution}

In this section, we present the brightness spatial distribution along the slit for the $3.3 \mu \mathrm{m}$ aromatic feature, the features in the 3.4-3.5 $\mu \mathrm{m}$ region, and the $\mathrm{HI}$ line at $3.74 \mu \mathrm{m}$. We also show the brightness spatial distribution of the continuum emission taken at adjacent wavelengths to the $3.3 \mu \mathrm{m}$ PAH feature. The continuum emission may be due to warm large-grain emission from the disk and/or stellar photospheric emission, as discussed in the following and in Sect. 5.

\subsubsection{Spatially extended $3.3 \mu \mathrm{m} \mathrm{PAH}$ feature}

Figure 2 shows the intensity profile along the slit of the $3.3 \mu \mathrm{m}$ $\mathrm{PAH}$ feature, the adjacent continuum, and the observed reference star (the assumed PSF) for the 4 sources where we detect the $3.3 \mu \mathrm{m}$ PAH feature. The $3.3 \mu \mathrm{m}$ emission feature has been corrected for the contribution of $\mathrm{Pf} \delta$, as described in Sect. 4.1.3.

In all objects the spatial extent of the PAH emission is significantly broader than that of the PSF. On the other hand, the continuum emission is unresolved, with the exception of HD 97048, where it is marginally resolved (see also Habart et al. 2004c).

To estimate the typical error on the measured full-width at half-maximum ( $F W H M)$ size of the PAH emission profiles, we do simulations as follows. For each target, we take the NACO data of a star with similar characteristics to the selected target, but with no PAH feature, and add to it a synthetic $\mathrm{PAH}$ feature emission profile with a given physical size convolved with the PSF and the same observed intensity. Then we process these synthetic image in the same way as we do for the observations, and the difference between the input and derived sizes gives us an estimation of the typical error. For our objects, the typical error in the measured $F W H M$ ranges between $10-20 \%$. 

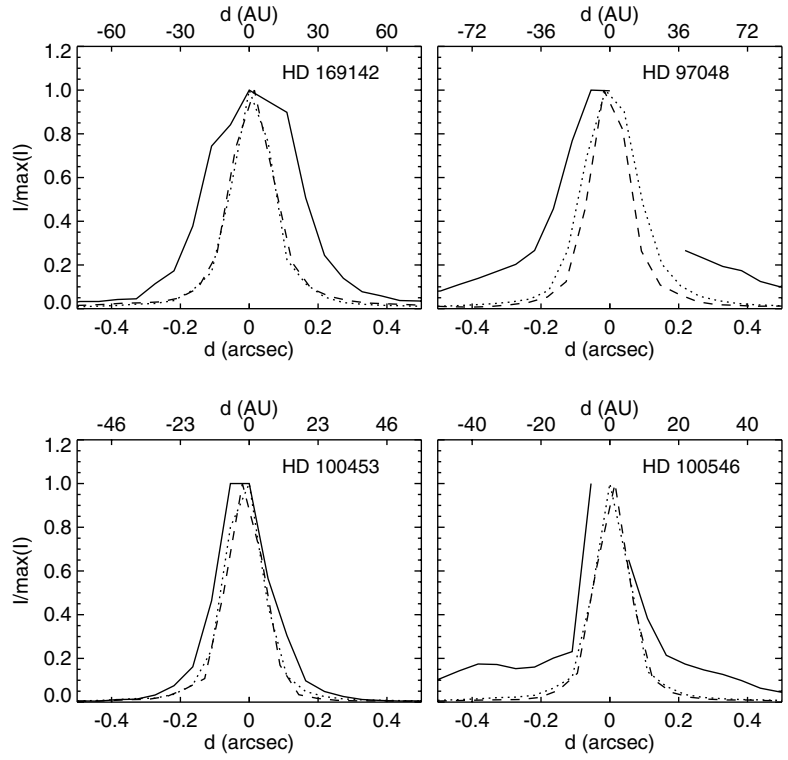

Fig. 2. Normalized spatial emission profiles of the $3.3 \mu \mathrm{m}$ PAH feature (adjacent continuum subtracted, solid lines) as a function of the distance from the star. Dotted and dashed lines show the intensity profile of the adjacent continuum and of the PSF, respectively. The scale on the lower axis shows the distance from the star in arcsec, and the upper axis the corresponding value in AU. For HD 97048, on one section of the slit and for distances from the star from 0 to $0.2^{\prime \prime}$, the NACO spectrum was noisy near the $3.3 \mu \mathrm{m}$ feature, and it was not possible to measure its brightness profile with sufficient accuracy. For HD 100546, we detected the presence of a gap in the innermost region $\left(R \leq 0.05-0.1^{\prime \prime}\right.$ or 5-10 AU); in this region, the spectrum at $3.3 \mu \mathrm{m}$ is in fact completely dominated by the contribution of $\operatorname{Pf} \delta$, and we do not see the PAH emission (see text for more details).

HD 169142. The PAH emission feature at $3.3 \mu \mathrm{m}$ is extended with an FWHM of $0.3^{\prime \prime}$ (or $43 \mathrm{AU}$ ). The feature is clearly visible above the continuum and is strong in the inner regions (radius $\leq 0.3^{\prime \prime}$ ), decreasing rapidly in the outer regions. For radius $>0.3^{\prime \prime}$, the feature is weak, so it is not possible to measure its brightness profile with sufficient accuracy.

HD 97048. The $3.3 \mu \mathrm{m}$ emission feature appears extended as in HD 169142. On one section of the slit and for distances from the star from 0 to $0.2^{\prime \prime}$, the NACO spectrum was noisy near the $3.3 \mu \mathrm{m}$ feature, so it was not possible to measure its brightness profile with sufficient accuracy. As in HD 169142, the $3.3 \mu \mathrm{m}$ feature is weak in the outer regions and given the uncertainties of the data, differences in the wings between the two objects are not significant. The continuum emission is spatially resolved in HD 97048 with an FHWM of $0.180^{\prime \prime}$ (or $32 \mathrm{AU})$; this indicates that in this system the continuum emission is mostly due to grain emission from the disk.

HD 100453. The $3.3 \mu \mathrm{m}$ feature emission is marginally resolved, with an FWHM of $0.18^{\prime \prime}$ (or $20 \mathrm{AU}$ ). In this object, the PAH emission feature decreases very rapidly in the outer zones. The spatial extension of the PAH emission is significantly smaller than the one observed in the two objects discussed previously. To quantitatively test our ability to resolve the PAH emission in this system, we used the simulations described above. When we simulated observations similar to those of HD 100453 with an input convolved size of $0.18^{\prime \prime}$, we found that the continuum substracted synthetic line profile fited the observed one very well (difference on the FWHM of $\lesssim 10 \%$ ) and concluded that the apparent small spatial extension seen in this system is real.

The rapid decrease in the $\mathrm{PAH}$ emission in this system could be due to low excitation conditions due to the later type (A9) of the central star.

HD 100546. The PAH emission spatial distribution in HD 100546 differs in several points from that found in the other objects. Firstly, we detect the presence of a gap in the innermost region $\left(R \leq 0.05-0.1^{\prime \prime}\right.$ or $\left.5-10 \mathrm{AU}\right)$. In this region, as discussed in Sect. 4.1.3, the spectrum at $3.3 \mu \mathrm{m}$ is in fact completely dominated by the contribution of $\operatorname{Pf} \delta$, so we do not see the PAH emission. This gap is also found for the emission of the additional weaker features in the 3.4-3.5 $\mu \mathrm{m}$ region (see Sect. 4.2.2), where there are no HI lines. It could be due to the lack of the carrier of the bands in the regions that are very near the star or to the fact that the emission of these features disappears completely in contrast to the continuum. But it could also result from the presence of a "physical hole", as suggested by the analysis of the ISO spectra of HD 100546 by Bouwman et al. (2003). In order to explain the amount of mid-IR dust emission and the observed crystalline features, Bouwman et al. (2003) propose that the disk should have a hole and a wall at about $10 \mathrm{AU}$. In the NACO data, there is no gap in the $3 \mu \mathrm{m}$ continuum emission, as expected in the case of a "physical hole". However, we cannot rule out, on the basis of our data, that the stellar photosphere contributes a major fraction of the $3 \mu \mathrm{m}$ flux. Higher spatial resolution data, combined with an accurate analysis of the spectral energy distribution (SED), are necessary to shed light on this very interesting object.

We also find that the $3.3 \mu \mathrm{m}$ emission feature at larger distances is stronger in HD 100546 than in the other objects. We can easily trace the $3.3 \mu \mathrm{m}$ emission feature to distances of $\sim 0.5 \operatorname{arcsec}$ or $50 \mathrm{AU}$ from the star. However, because of the presence of the gap, it is very hard to assess whether the central region is more extended in the PAH band than in the continuum, and it is not possible to determine the FWHM size of the PAH emission band. Nevertheless, we note that our NACO data are consistent with an $F W H M$ of $\sim 0.2^{\prime \prime}$ (20 AU) recently measured from ISAAC spectroscopic observations (Geers et al. 2004).

\subsubsection{Spatial extension of the features in the 3.4-3.5 $\mu \mathrm{m}$ region}

We now look at the spatial emission distribution of the features in the 3.4-3.5 $\mu \mathrm{m}$ region observed in two of our objects (HD 97048, HD 100546) and compare it to that of the $3.3 \mu \mathrm{m}$ PAH feature.

Figure 3 shows the intensity profile of the strongest feature at $3.53 \mu \mathrm{m}$ attributed to diamonds, observed in HD 97048. The intensity profile of the other strong diamond feature at $3.43 \mu \mathrm{m}$ is similar. The derived FWHM is of $0.23^{\prime \prime}$ (41 AU) in both the 3.43 and $3.53 \mu \mathrm{m}$ features (see Habart et al. 2004c). 

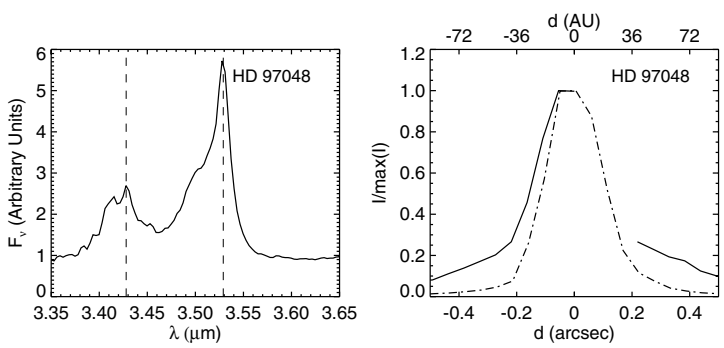

Fig. 3. Left panel: spectrum of HD 97048 in the 3.35-3.65 $\mu \mathrm{m}$ range obtained for distances from the star $\leq 1^{\prime \prime}$. The dotted vertical lines show the strong diamond feature positions. Right panel: normalized spatial emission profiles of the $3.3 \mu \mathrm{m}$ PAH features (adjacent continuum subtracted, solid lines) and the $3.53 \mu \mathrm{m}$ diamond feature (adjacent continuum subtracted, dotted-dashed line) as a function of the distance from the star. The scale on the lower axis shows the distance from the star in arcsec, that on the upper axis the corresponding value in $\mathrm{AU}$.
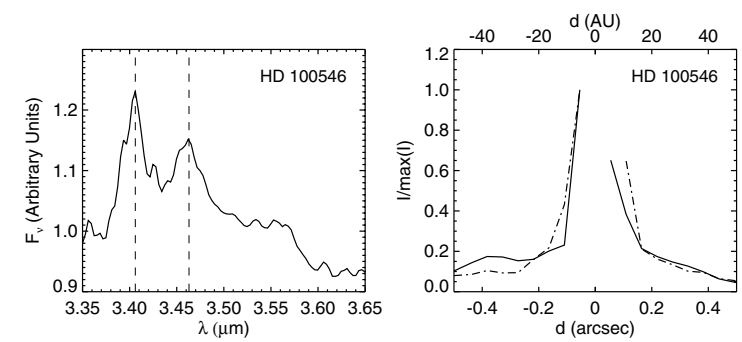

Fig. 4. Left panel: spectrum of HD 100546 in the 3.35-3.65 $\mu \mathrm{m}$ range obtained for distances from the star going from 0.2 to $0.6^{\prime \prime}$. The dotted vertical lines show the positions of the additional features. Right panel: normalized spatial emission profiles of the $3.3 \mu \mathrm{m}$ PAH features (adjacent continuum subtracted, solid lines) and the $3.4 \mu \mathrm{m}$ feature (adjacent continuum subtracted, dotted-dashed line) as a function of the distance from the star. The scale on the lower axis shows the distance from the star in arcsec, that on the upper axis the corresponding value in AU.

Also, by comparing the spatial extension of these features to that of the continuum and of the PAH at $3.3 \mu \mathrm{m}$, we find that the diamond emission is extended more than the continuum but less than that of the PAHs. This is roughly consistent with the idea that the diamond carrier of the $3 \mu \mathrm{m}$ features observed in HD 97048 have sizes between those of large grains and of PAHs, as suggested by detailed analysis of the ISO spectrum (see e.g. Van Kerckhoven et al. 2002; Sheu et al. 2002; Jones et al. 2004). Finally, we note that recent AO observations at the Subaru telescope of Elias 1, which shows the same diamond bands at 3.43 and $3.53 \mu \mathrm{m}$ as HD 97048, show similar results (M. Goto, private communications). The observed $F W H M$ of the diamond features is similar to the one derived here for HD 97048, and the PAH emission is also found to be spatially more extended than for the diamonds.

Figure 4 shows the intensity profile of the strongest additional feature at $3.4 \mu \mathrm{m}$ observed in HD 100546. As for the PAH $3.3 \mu \mathrm{m}$ feature, there is evidence of a gap in the innermost regions. Also, the emission of the "satellite" feature is extended as the PAH emission. This is roughly consistent with the idea that the carrier of these features is comparable in sizes to the PAHs and compatible with the two identifications of these

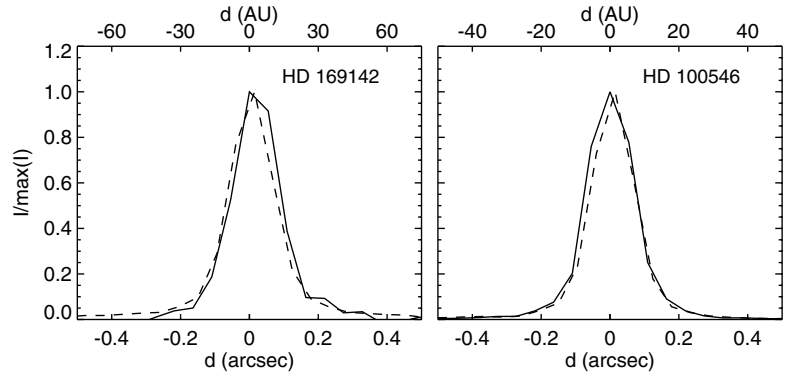

Fig. 5. Normalized spatial emission profiles of the HI line at $3.74 \mu \mathrm{m}$ (adjacent continuum subtracted, solid lines) as function of the distance from the star. Dashed lines show the intensity profile of the PSF. The scale on the lower axis shows the distance from the star in arcsec, that on the upper axis the corresponding value in AU.

features suggested previously, i.e., aliphatic C-H stretches in methyl or ethyl side-groups attached to PAHs or nanodiamonds with sizes of a few nm.

\subsubsection{Spatial extension of the hydrogen emission lines}

Finally, we look at the spatial extension of the HI Pf $\gamma$ recombination line at $3.74 \mu \mathrm{m}$. Figure 5 shows the intensity profile of Pf $\gamma$ observed in two of our objects (HD 169142, HD 100546) where we detected it clearly. The HI emission line is not resolved. It originates in ionized material very close to the star, possibly the ionized gaseous disk or matter accreting from the inner disk onto the star.

\section{Comparison to model predictions}

In this section, we compare the observed spatial distribution of the $3.3 \mu \mathrm{m}$ PAH feature to predictions of a disk model that includes PAHs and very small grains in addition to large grains. These models (Habart et al. 2004a) reproduce the strength of the most commonly observed PAH features (i.e., 3.3, 6.2, 7.7, $11.3 \mu \mathrm{m})$ in many HAeBe stars.

\subsection{Description of the model}

In Habart et al. (2004a), we computed models of disks heated by irradiation from the central star, in hydrostatic equilibrium in the vertical direction, and with dust and gas mixed well (flared disks). We used a 2-layer model to calculate the disk structure and implemented a 1D radiative transfer code to compute the emerging spectrum.

The models are appropriate for disks that are optically thick to the stellar radiation. This is generally the case for disks around pre-main-sequence stars, up to very large radii. For simplicity, we limit ourselves here to a disk with $M_{\mathrm{d}} \sim 0.1 M_{\odot}$, an inner radius of $R_{\text {in }}=0.3 \mathrm{AU}$ (close to the BG evaporation radius), and an outer radius of $R_{\mathrm{d}}=300 \mathrm{AU}$. The surface density profile is $\Sigma=\Sigma_{0} \times\left(r / R_{i}\right)^{-1}$ with $\Sigma_{0}=2 \times 10^{3} \mathrm{~g} \mathrm{~cm}^{-2}$.

The dust is a mixture of large grains in thermal equilibrium, transiently heated small grains, and PAHs. The big grains consist of graphite and silicates with optical constants from Draine (1985); they have an MRN size distribution $\left(n(a) \propto a^{-3.5}\right)$ 
between a minimum and a maximum radius $a_{l}=0.01 \mu \mathrm{m}$ and $a_{u}=0.74 \mu \mathrm{m}$ for silicates and $a_{l}=0.01 \mu \mathrm{m}$ and $a_{u}=$ $0.36 \mu \mathrm{m}$ for graphite. To this grain population, we add a tail of the very small graphite particles with the same size distribution $\left(n(a) \propto a^{-3.5}\right)$ between $a_{l}=10 \AA$ and $a_{u}=100 \AA$. Optical constants are from Draine (1985).

For the population of PAHs, the absorption cross section is from Li \& Draine (2001). In the ISM, PAHs are made up of a few tens up to a few hundreds of carbon atoms; for reasons of simplicity, we take one PAH size in our model, $N_{\mathrm{C}}=100$. The hydrogen to carbon ratio is $\mathrm{H} / \mathrm{C}=f_{\mathrm{H}} \times\left(6 / N_{\mathrm{C}}\right)^{0.5}$ (case of compact symmetric PAHs, see Omont 1986) with $f_{\mathrm{H}}-$ the hydrogenation fraction of the molecule - equal to 1 (i.e., essentially fully hydrogenated PAHs). With respect to the charge, to keep the model simple we ignore that probably in disks we have a mixture of neutral and charged PAHs and assume that PAHs are neutral. Finally, we consider that in a strong FUV radiation field, photo-destruction of PAHs will occur during multiphoton events where PAHs absorb an energy of more than $21 \mathrm{eV}$ in a time shorter than the cooling time. In our model, we assume that PAHs are destroyed when the probability that they are at a temperature $T \geq T_{\text {evap }}\left(T_{\text {evap }}=2000 \mathrm{~K}\right)$ exceeds a critical value $p_{\text {evap }}\left(p_{\text {evap }}=10^{-8}\right)$ (for more details see Habart et al. 2004a). The effect of these assumptions is briefly discussed in Sect. 5.4.

Finally, the silicate abundance in dust is $[\mathrm{Si} / \mathrm{H}]=3 \times 10^{-5}$, and the total carbon abundance in dust is $[\mathrm{C} / \mathrm{H}]=2.22 \times 10^{-4}$. Of this, $23 \%$ are in PAHs, $10 \%$ in VSGs and $67 \%$ in large grains.

The model predicts an infrared spectral energy distribution showing PAH features at 3.3, 6.2, 7.7, and $11.3 \mu \mathrm{m}$ that are clearly visible above the continuum emission mostly due to warm large grains (see Fig. 3 in Habart et al. 2004a). In Habart et al. (2004a), we compared the model results to observations from ISO and ground-based telescopes of some thirty HAeBe stars, including all our targets except CQ Tau. Considering the uncertainty due to our disk description and assumed dust model, we concluded that the model can account for the strength of the most commonly observed PAH features (i.e., $3.3,6.2,7.7,11.3 \mu \mathrm{m}$ ) in most of the objects with flared disks.

For geometrically flat disks, PAH features are predicted to be very weak, even when PAHs with standard properties are present on the disk surface. This agrees with the obervations showing that in objects with no evidence of a flared disk (group II) no (or weak) PAH features have been detected.

\subsection{Predicted spatial distribution}

The model predicts that the PAH emission is much more spatially extended than the adjacent continuum emission, as shown in Fig. 6. Figure 6a plots the cumulative intensity of the PAH feature and continuum emission at $3.3 \mu \mathrm{m}$ as a function of the radius. The continuum reaches $50 \%$ of its intensity at a very small radius (about $2 \mathrm{AU}$ ), while the feature does so at larger radii (about $30 \mathrm{AU}$ ). This behavior basically reflects the different excitation mechanism of PAHs, which are transiently heated by individual UV photons, and of the continuum, which
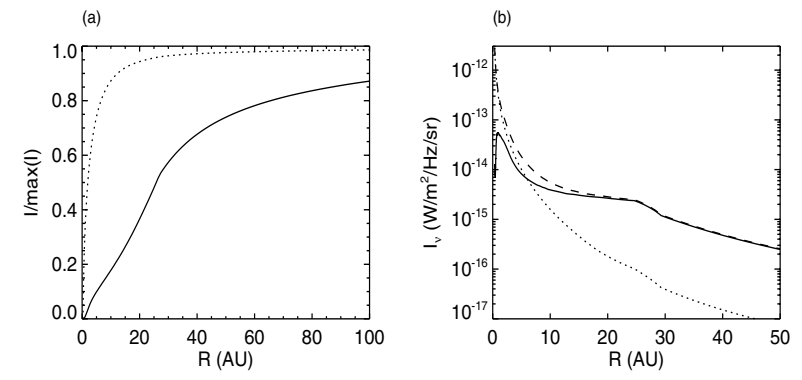

Fig. 6. Disk model predictions. Panel a): cumulative intensity of the PAHs feature (continuum subtracted, solid line) and adjacent continuum (dotted line) at $3.3 \mu \mathrm{m}$ as a function of the projected radius. Panel b): predicted surface brightness profiles at the peak of the $3.3 \mu \mathrm{m}$ feature (no continuum subtracted, dashed line) and in the adjacent continuum (dotted line). We also show the $3.3 \mu \mathrm{m}$ feature continuum subtracted as a solid line. The disk is heated by a typical HAe star with effective temperature $T_{\text {eff }}=10500 \mathrm{~K}$, luminosity $L_{\star}=32 L_{\odot}$ and mass $M_{\star}=2.4 M_{\odot}$. The disk is flared, it has a mass of $0.1 M_{\odot}$, an inner radius of $0.3 \mathrm{AU}$, and an outer radius of $300 \mathrm{AU}$. The star/disk system is seen face-on at a distance of $150 \mathrm{pc}$.

is dominated by the emission of big grains in thermal equilibrium with the radiation field. Only big grains located very near the star are hot enough to emit at $3 \mu \mathrm{m}$, whereas PAHs farther away can also be excited and emit in the $3.3 \mu \mathrm{m}$ feature. However, the $3.3 \mu \mathrm{m}$ feature is also expected to be stronger near the star, since the emission of PAHs roughly scales with the intensity of the FUV radiation field. Moreover, among the PAH features, the $3.3 \mu \mathrm{m}$ one is the least extended spatially. As discussed in Habart et al. (2004a), as well for transiently heated particles, the features at short wavelengths are in fact stronger in the inner part of the disk (where PAHs are hotter because multiphoton events occur where the radiation field is highest), decreasing rapidly in the outer cold part. On the other hand, the features at longer wavelengths are more extended.

Figure $6 \mathrm{~b}$ shows the surface brightness profiles of the $3.3 \mu \mathrm{m}$ feature and the adjacent continuum. As expected from Fig. 6a, the PAH emission feature is much more extended than the adjacent continuum. Finally, it is interesting to note that for the formalism adopted here, we find that very near the star ( $R<1-2$ AU) PAHs are mostly photoevaporated, as shown by the inner gap seen in the $3.3 \mu \mathrm{m}$ feature continuum subtraced emission profile (see Fig. 6b). At $R \sim 15 \mathrm{AU}$, about $50 \%$ of PAHs are photo-evaporated and for $R>20$ AU this fraction goes to zero.

\subsection{Comparison with the observations}

Figure 7 shows the intensity profile of the $3.3 \mu \mathrm{m}$ feature (continuum subtracted) for each of our objects showing the $3.3 \mu \mathrm{m}$ feature obtained by convolving the computed intensity with a fit to the corresponding observed PSF (i.e., the average between the PSF profiles observed on both star sides). For each object, we computed a model with the suitable stellar parameters given in Table 1. The disk and dust parameters are the same as in the previous discussed model shown in Fig. 6 and as described in Sect. 5.1. The star/disk system is assumed to be seen face-on at the distance of the object. Figure 7 also shows 

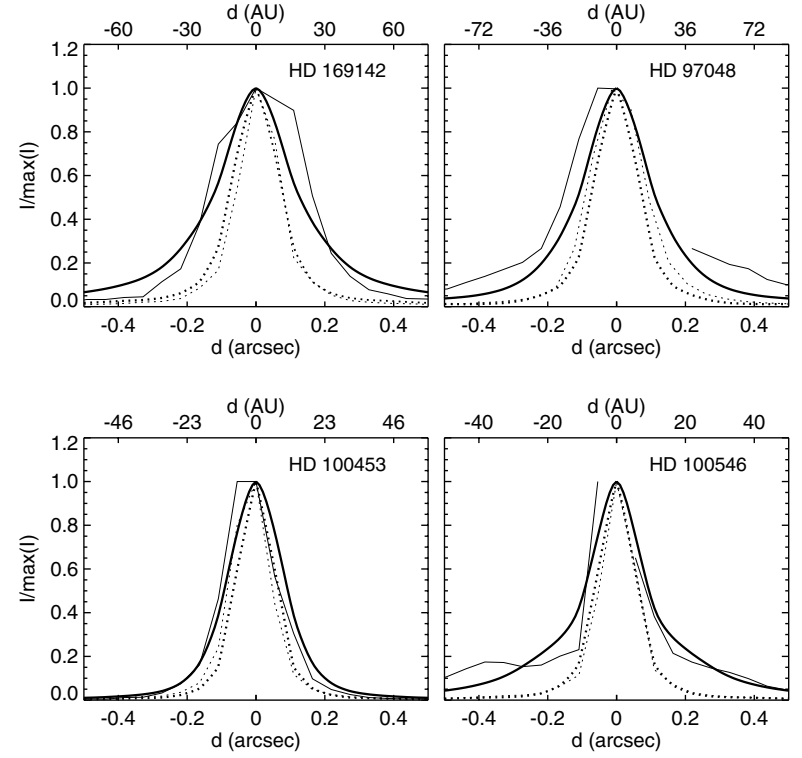

Fig. 7. Comparison of model predictions and observations. Normalized spatial emission profiles of the $3.3 \mu \mathrm{m}$ PAH feature (continuum subtracted, solid lines) and of the adjacent continuum (dotted lines) as a function of the distance from the star. The thick and thin lines show the predicted and observed spatial emission profiles, respectively. For each object, the predicted emission profiles have been convolved with a fit of the corresponding observed PSF. The star/disk system is assumed to be seen face-on at the distance of the object. In each panel, the dashed line shows the normalized spatial emission profile of the PSF. The scale on the lower axis shows the distance from the star in arcsec, that on the upper axis the corresponding value in $\mathrm{AU}$.

the predicted intensity profile of the adjacent continuum, which is the sum of the computed disk continuum emission (mostly due to warm large grains) and of the photosphere stellar emission at $3 \mu \mathrm{m}$, each convolved with the fit of the observed PSF. The stellar contribution to the continuum emission at $3 \mu \mathrm{m}$ is typically about $30 \%$ for our stars with spectral type of B9-A5, while it dominates for the later spectra type stars. The exact value of the stellar contribution depends not only on the stellar properties but also on disk parameters. Note, however, that the continuuum subtracted intensity profile of the PAH emission is independent of them.

By comparing the predictions to the observations, we find that, as observed, the predicted $3.3 \mu \mathrm{m}$ PAH emission is significantly broader than that of the adjacent continuum and its predicted $F W H M$ is very close to the observed one (differences $\leq 20 \%$, see Fig. 7). For some objects, there are intringuing differences between model predictions and observations in the wings, which are however generally within the uncertainties of the data. On the contrary, the adjacent continuum emission is not resolved or is only slighlty broader than the PSF. This also agrees with the observations. We take the general agreement between observations and predictions as strong support for the physical pictures underlying our model.

It must be emphasized that there are several complications that we have neglected. The most obvious is that we have assumed that PAHs can be characterized by a single size, hydrogenation, and charge state. This is unlikely to be the case and is discussed in the next section. However, as also discussed in the following, the predicted brightness profiles of the $3.3 \mu \mathrm{m}$ feature for different PAHs properties are similar in the inner regions and show only significant differences at large radii, $R \gtrsim 40 \mathrm{AU}$

\subsection{Discussion}

Our results allow us to locate the PAH emission in the disk, to show that the emission of the PAHs is spatially more extended than that of adjacent continuum, and to prove beyond doubt that the energetic $3.3 \mu \mathrm{m}$ PAH feature takes its origin in the inner disk regions. It is interesting to note that the spatial extension of the $3.3 \mu \mathrm{m}$ feature is much smaller than those observed for PAH features at longer wavelength (i.e., 8.6, 11.3, $12.7 \mu \mathrm{m}$ ), which are extended on a scale of (several) $100 \mathrm{AU}$ in HD 97048 and HD 100546 (van Boekel et al. 2004). This is consistent with the model results, which showed how among the PAH features in disks the $3.3 \mu \mathrm{m}$ one is the least extended spatially (see Sect. 5.2).

Our results have the interesting implication that PAHs survive photo-evaporation or coagulation processes even if these processes should be in principle important in the inner disk, where the UV flux and the gas density are very high. Nevertheless, processes such as photo-evaporation must significantly affect the abundance of PAHs. For the formalism adopted here, we find that in the $R<20$ AU region about $60 \%$ of PAHs (with $N_{\mathrm{C}}=100$ ) are photo-evaporated. But PAH evaporation is a complex process that increases rapidly with $\chi$ and for smaller PAHs, so it is difficult to make quantitative estimates. Alternatively, it is possible that continuous replenishment of PAHs via sublimation of icy mantles (in which interstellar PAHs may have condensed during the dense molecular cloud phase) or by accreting carbon atoms and/or ions from the gas (see, e.g., Allain et al. 1996) maintain the PAHs population throughout the disk. This is a very interesting point and future detailed comparison with theoretical calculations will certainly add important information to the studies of the processes affecting the size of grains in disks.

In the inner disk regions, processes such as ionization or de-hydrogenation are also expected to be important (because of the strong UV field) and to modify the PAH properties. One expect PAH properties to vary as a function of the radius and depth in the disk. For example, PAHs are likely to be more positively ionized in the inner disk regions. Moreover, processes such as photo-evaporation or coagulation could affect the size of PAHs. Therefore, it is unlikely that PAHs are characterized by a single charge state, size, or hydrogenation parameter, as assumed in our model. In order to get some insight into the specific PAH properties, one needs detailed comparison between the observations not only of the intensity of the various features, as in Habart et al. (2004a), but also of the brightness profiles at large radii, $R \gtrsim 40 \mathrm{AU}$, where different PAHs have very different emission (e.g., Figs. 6 and 7 in Habart et al. 2004a). Unfortunately, our NACO data in the wings are not of sufficient enough quality to be used in this context, but we want to 
emphasize that future higher sensitive, spatially resolved spectroscopic data at $\sim 3 \mu \mathrm{m}$ have the potential capability of constraining the PAH properties and of studying their evolution.

\section{Summary and conclusion}

We have presented NACO/VLT spectroscopic observations in the $3 \mu \mathrm{m}$ region of a sample of HAeBe stars. These data allow us, for the first time, to spatially resolve the inner disk emission in the bands of small transiently heated particles and thus provide a benchmark for testing not only the dust properties within the disk but also the inner disk properties. Our main results can be summarized as follows.

1. We spatially resolved the $3.3 \mu \mathrm{m}$ PAH emission for all the sources where this feature is detected. We found that the PAH emission is significantly broader than that of the adjacent continuum. On the other hand, the continuum emission remains unresolved, with the exception of HD 97048 where it is marginally resolved.

2. We also spatially resolved the emission in the additional carbon dust features in the 3.4-3.5 $\mu \mathrm{m}$ region detected in two of our sources (HD 100546 and HD 97048). In HD 97048, the emission of the strong features at 3.43 and $3.53 \mu \mathrm{m}$ attributed to diamond surface $\mathrm{C}-\mathrm{H}$ stretching modes is extended more than the continuum emission but less than the PAH emission. This is roughly consistent with the idea that the diamond carriers of the $3 \mu \mathrm{m}$ features observed in this object have sizes that are intermediate between those of large grains and of PAHs, as suggested by detailed analysis of the ISO spectrum (Van Kerckhoven et al. 2002; Sheu et al. 2002; Jones et al. 2004). On the other hand, the emission of the weaker features at 3.4 and $3.46 \mu \mathrm{m}$ detected in HD 100546 is extended as the PAH emission. These features could be due to aliphatic $\mathrm{C}-\mathrm{H}$ stretches in methyl or ethyl side-groups attached to PAHs or nanodiamonds.

3. In HD 100546, we detected the presence of a gap in the innermost regions ( $R \leq 0.05-0.1^{\prime \prime}$ or $\left.5-10 \mathrm{AU}\right)$ in both the aromatic and weak additional features. This gap could be due to the lack of the carrier of the bands in the regions very near the star or to the fact that the emission of these features disappears completely in contrast to the continuum. But it could also result from the presence of a "physical hole", as suggested by the analysis of the ISO spectra of HD 100546 by Bouwman et al. (2003). Higher spatial resolution data combined with accurate analysis of the SED are necessary to shed light on this very interesting object.

4. We detected the HI Pf $\gamma$ recombination line clearly in practically all stars; the line is not spatially resolved, and probably originates from ionized material very close to the star, possibly the ionized gaseous disk or matter accreting from the inner disk onto the star.

5. We compared the observed spatial distribution of the $3.3 \mu \mathrm{m}$ PAH feature and the adjacent continuum to the predictions of a disk model that includes transiently heated small grains and PAHs, in addition to large grains (Habart et al. 2004a). The model predicts that, as observed, the
$3.3 \mu \mathrm{m}$ PAH emission feature is significantly broader than that of the adjacent continuum and that $50 \%$ of its integrated intensity comes from a radius $R<30$ AU. We find very good agreement of the predicted brightness profiles with our observations. This strong supports the physical pictures underlying our model and show that the $3.3 \mu \mathrm{m}$ PAH feature comes mostly from the inner disk regions.

6. Finally, our results have the interesting implication that PAHs survive photo-evaporation or coagulation processes even if these processes are in principle important in the inner disk, where the UV flux and the gas density are very high. Future detailed comparison with theoretical calculations will certainly add important information to the studies of the processes affecting the size of grains in disks.

\section{References}

Acke, B., \& van den Ancker, M. E. 2004, A\&A, 426, 151

Allain, S., Bouvier, J., Prosser, C., Marschall, L. A., \& Laaksonen, B. D. 1996, A\&A, 305, 498

Augereau, J. C., Lagrange, A. M., Mouillet, D., \& Ménard, F. 2001, A\&A, 365, 78

Blades, J. C., \& Whittet, D. C. B. 1980, MNRAS, 191, 701

Bouwman, J., De Koter, A., Dominik, C., \& Waters, L. B. F. M. 2003, A\&A, 401, 577

Brooke, T. Y., Tokunaga, A. T., \& Strom, S. E. 1993, AJ, 106, 656

Draine, B. T. 1985, ApJS, 57, 587

Geballe, T. R., Lacy, J. H., Persson, S. E., McGregor, P. J., \& Soifer, B. T. 1985, ApJ, 292, 500

Geers, V. C., Augereau, J.-C., Pontoppidan, K. M., et al. 2004, Proc. of the ESO Workshop on High Resolution Infrared Spectroscopy in Astronomy, ed. H.-U. Kaufl, R. Siebenmorgen, \& A. Moorwood

Grady, C. A., Polomski, E. F., Henning, T., et al. 2001, AJ, 122, 3396

Guillois, O., Ledoux, G., \& Reynaud, C. 1999, ApJ, 521, L133

Habart, E., Natta, A., \& Krügel, E. 2004a, A\&A, 427, 179

Habart, E., Boulanger, F., Verstraete, L., Walmsley, C. M., \& Pineau Des Forêts, G. 2004b, A\&A, 414, 531

Habart, E., Testi, L., Natta, A., \& Carbillet, M. 2004c, ApJ, 614, L129

Habing, H. J. 1968, Bull. Astron. Inst. Netherlands, 19, 421

Hummer, D. G., \& Storey, P. J. 1987, MNRAS, 224, 801

Joblin, C., Tielens, A. G. G. M., Allamandola, L. J., \& Geballe, T. R. 1996, ApJ, 458, 610

Jones, A. P., d'Hendecourt, L. B., Sheu, S. Y., et al. 2004, A\&A, 416, 235

Jourdain De Muizon, M., D’Hendecourt, L. B., \& Geballe, T. R. 1990a, A\&A, 235, 367

Jourdain De Muizon, M., D’Hendecourt, L. B., \& Geballe, T. R. 1990b, A\&A, 235, 367

Li, A., \& Draine, B. T. 2001, ApJ, 554, 778

Meeus, G., Waters, L. B. F. M., Bouwman, J., et al. 2001, A\&A, 365, 476

Natta, A., Prusti, T., Neri, R., et al. 2001, A\&A, 371, 186

Omont, A. 1986, A\&A, 164, 159

Sheu, S. Y., Lee, I. P., Lee, Y. T., \& Chang, H. C. 2002, ApJ, 581, L55

Thé, P. S., Tjin, H. R. E, Steenman, H., \& Wesselius, P. R. 1986, A\&A, 155,347

van Boekel, R., Waters, L. B. F. M, Dominik, C., et al. 2004, A\&A, 418, 177

Van Kerckhoven, C., Tielens, A. G. G. M., \& Waelkens, C. 2002, A\&A, 384, 568

Van Kerckhoven, C. 2002, Ph.D Thesis, University of Leuven 